Palabra Clave (La Plata), Octubre 2018, vol. 8, n 1, e059. ISSN 1853-9912

Universidad Nacional de La Plata.

Facultad de Humanidades y Ciencias de la Educación.

Departamento de Bibliotecología

\title{
La migración de Alephino a Koha: el caso de la biblioteca de Espigas
}

\author{
Melina Alicia Cavalo \\ Centro de Estudios Espigas, Tarea-Instituto de Investigaciones sobre el \\ Patrimonio Cultural, Universidad Nacional de San Martín, Argentina \\ melinaacavalo@gmail.com

\section{Ramiro Federico Uviña} \\ Universidad de Buenos Aires. Facultad de Filosofía y Letras Universidad \\ Nacional de Tres de Febrero. Instituto de Investigación, Arte y Cultura, \\ Argentina \\ ramirouvia@gmail.com
}

Cita sugerida: Cavalo, M. A. y Uviña, R. F. (2018). La migración de Alephino a Koha: el caso de la biblioteca de Espigas. Palabra Clave (La Plata), 8(1), e059. https://doi.org/10.24215/18539912e059

Recibido: 14 de mayo de 2018 - Aceptado: 29 de octubre de 2018 - Publicado: 31 de octubre de 2018

(c) (1) (3)(2) Esta obra está bajo licencia Creative Commons Atribución-NoComercial-CompartirIgual 4.0 Internacional http://creativecommons.org/licenses/by-nc-sa/4.0/deed.es_AR 


\title{
La migración de Alephino a Koha: el caso de la biblioteca de Espigas
}

\section{The migration from Alephino to Koha: the case of the Espigas library}

\author{
Melina Alicia Cavalo \\ Centro de Estudios Espigas, Tarea-Instituto de \\ Investigaciones sobre el Patrimonio Cultural, Universidad \\ Nacional de San Martín, Argentina \\ melinaacavalo@gmail.com
}

Ramiro Federico Uviña

Universidad de Buenos Aires. Facultad de Filosofía y Letras

Universidad Nacional de Tres de Febrero. Instituto de

Investigación, Arte y Cultura, Argentina

ramirouvia@gmail.com

\section{Resumen:}

En el presente trabajo se describe el proceso de migración de registros bibliográficos y de existencias desde el sistema integrado de gestión bibliotecaria Alephino a Koha, a partir de la experiencia desarrollada en la biblioteca del Centro de Estudios Espigas, Tarea-IIPC (Instituto de Investigaciones sobre el Patrimonio Cultural), UNSAM (Universidad Nacional de General San Martín). Este artículo centra su foco en el mapeo de los campos y subcampos entre los registros en formato bibliográfico MaRC21 y de los registros de existencia, diferentes entre ambos sistemas, ya que para el resto del proceso de migración se utilizaron herramientas conocidas en la bibliografía existente y/o documentación oficial (MarcEdit, Wiki de Koha, etc.). El resultado del proceso emprendido consistió en la migración sin pérdida de información del total de los registros de manera exitosa. A modo de conclusión se presenta la posición de los autores respecto a la importancia en el proceso de migración de la cooperación entre profesionales y el empleo de software libre.

Palabras ClaVe: Migración, Software libre, Mapeo.

\section{ABstRACT:}

In the present work we intend to describe the process of migration of bibliographic and item records between the integrated library systems Alephino to Koha, from the case carried out in the library of the Centro de Estudios Espigas, Tarea-IIPC (Instituto de Investigaciones sobre el Patrimonio Cultural), UNSAM (Universidad Nacional de General San Martín). The methodology used focused on the mapping of fields and subfields between the records in the MaRC21 bibliographic format, and the difference in the existence records between both systems. The result consisted of the successful in the migration without loss of information of the total of the records. In conclusion, the position of the authors regarding the importance in the migration process of cooperation between professionals and the use of free software is presented.

KEYwORDS: Migration, Free software, Crosswalk.

\section{INTRODUCCIÓN}

La Fundación Espigas nace en 1993 como una ONG sin fines de lucro (con personería jurídica), con el objetivo de organizar y sostener un Centro de Documentación para la Historia de las Artes Visuales en la Argentina, disponible para la consulta pública en general. La misión del Centro es reunir, preservar y hacer accesible la memoria material y digital referida a la historia del arte argentino y latinoamericano, cuyo acervo se compone por un volumen estimado de 300.000 recursos distribuidos en tres corpus documentales: biblioteca, hemeroteca y archivo. A partir de 2017, por medio de un convenio con la Universidad Nacional de General San Martín (UNSAM), se crea el Centro de Estudios Espigas en el marco de Tarea-IIPC (Instituto 
de Investigaciones sobre el Patrimonio Cultural), con el objetivo de gestionar el acervo de Espigas y sostener la continuidad de su misión.

En este contexto se llevó a cabo, en primera instancia, un relevamiento de los procedimientos y los softwares empleados para la gestión de los recursos del acervo archivístico y bibliotecológico de la institución. El sistema empleado como base de datos en ese momento era Alephino, complementado por listados de recursos archivísticos publicados en la página web y un software diseñado ad hoc para las fotografías del acervo. Entre la documentación existente que fue relevada en el Centro, se encontró información de una migración anterior de registros desde el programa Winisis en la cual, a pesar de los trabajos de mapeo y revisión entre CEPAL y MaRC21, inevitablemente se habían detectado casos con pérdida de información.

Alephino es un ILS (Integrated Library System) perteneciente a la empresa ExLibris, al cual es posible suscribir a los módulos operativos de manera aislada. En el caso de Espigas, el sistema solo contemplaba el módulo de catalogación y el OPAC (Online Public Access Catalog), es decir, que no eran considerados los de control de autoridades, usuarios y circulación entre otros.

Frente a la renovación del contrato de soporte técnico anual para Alephino, en marzo de 2017 la nueva coordinación bibliotecológica de Espigas evaluó las ventajas y desventajas del sistema y estableció como política a futuro el empleo de software libre y de código abierto. Siguiendo la línea de trabajo de la biblioteca central de la UNSAM, se decidió adoptar el ILS Koha configurándolo para las áreas de biblioteca y hemeroteca y diferenciándolo del sistema seleccionado para el material de archivo. Para ello se convocó a un consultor externo que, junto con la responsable del área, se encargaron de planificar la migración de los 97.832 registros disponibles en Alephino. Debe mencionarse, que el proyecto inicial de 6 meses se debió concretar en 40 días, ya que el tiempo apremiaba frente al vencimiento de la suscripción anual al soporte de Alephino.

Cabe destacar que, a diferencia de muchas bibliotecas de Argentina, la biblioteca ya utilizaba un sistema integrado de gestión bibliotecaria, Alephino, en el que disponía de registros bibliográficos y de existencias catalogados en MaRC21. Y si bien se utilizó este formato durante los 24 años de existencia de la institución, al llevar adelante el proceso de migración, se identificaron 39 catalogadores y se detectó en los registros existentes poca consistencia en lo relativo a los parámetros de catalogación y su utilización.

Por otro parte, interesa resaltar que, si bien en la bibliografía especializada podemos encontrar trabajos sobre las migraciones entre sistemas que emplean el formato bibliográfico MaRC21 y otros, se dispone de escasa documentación sobre trabajos realizados con el sistema Alephino.

En el caso del presente artículo se pretende dar cuenta del proceso de migración de registros bibliográficos y de existencias desde el sistema integrado de gestión bibliotecaria Alephino a Koha. Para ello, se desarrolla la metodología empleada y se describen las particularidades que hacen a la migración de un sistema integrado de gestión bibliotecaria privativo - del cual se posee escasa a nula cantidad de información-, a un sistema integrado libre -del cual se posee una cantidad de recursos extraordinaria-, con las particularidades del caso de la biblioteca de la Fundación Espigas. Es por esta última razón que para la elección del software se privilegió el empleo del software libre y el trabajo cooperativo, transitando recorridos ya probados: el Koha es un sistema integrado de gestión bibliotecaria de reconocido éxito en el ámbito nacional y utilizado en instituciones afines / asociadas (biblioteca central de la Universidad Nacional de San Martín).

\section{Procesos realizados en la migración}

Ante el panorama previamente descripto se planificó realizar la migración de los registros existentes sin efectuar una limpieza de datos: migrando los datos tal y como estaban en el Alephino y poniendo el énfasis en que no se produjera ningún tipo de pérdida de información. Como se ha mencionado más arriba, una de las ventajas es que Alephino contempla tanto el estándar MaRC21 bibliográfico y de existencias y utiliza la mayor parte de los campos existentes en dichos formatos, a diferencia de Koha que solo posee algunos datos del formato de existencias. En esta parte del proceso, surgió un conflicto al momento de encontrar 
la relación entre los datos de estos estándares en ambos softwares ya que, si bien el contrato con el soporte técnico ExLibris estaba vigente al momento de realizar la migración, no fue posible obtener una respuesta precisa sobre qué campo utilizaba Alephino para vincular las tablas de registros bibliográficos con los registros de existencias.

Haciendo un barrido bibliográfico, se encontró que la experiencia más próxima a la migración de Alephino a Koha tuvo lugar en la Luleå Tekniska Universitet de Suecia en dónde realizaron el proceso de migración desde Aleph a Koha (How to migrate from, s.f.). Sin embargo, Aleph tiene una funcionalidad que no estaba disponible en la versión de Alphino que era utilizada en Espigas, que es la de exportar los registros en marcxml. Por lo tanto, luego de efectuar diversas pruebas y realizar una consulta informal a una colega, quien transitaba un camino parecido en su desarrollo profesional con Alephino hacia un sistema de biblioteca digital (Rus Mata y Plaza, 2017), se llegó a la siguiente solución ante el problema: emplear el campo 001 de los registros bibliográficos en conjunción con el BIB, subcampo "s" de existencias para poder recuperar la relación entre bibliográficos y existencias, que es la forma en la cual se vinculan los registros bibliográficos con los de existencias en el sistema Alephino: utiliza los 001 de los registros bibliográficos y los BIB\$s para vincular ambas tablas.

\subsection{Migración de datos}

Una vez resuelto este inconveniente fue necesario revisar el modo de reformatear los datos de Alephino para trasladarlos a Koha. En virtud del análisis de las posibilidades de migración se observó que la única opción viable desde Alephino era generar reportes o informes como archivos de texto en formato .exp (para más detalles de cómo se exportaba la información en este archivo ver figura 1). Este archivo contenía prefijos que facilitaban su conversión y, a primera vista, parecía ser posible, luego de un mínimo reformateo, su adecuación a un archivo .mrk de manera sencilla. Un archivo .mrk es el utilizado por MaRCEdit para trabajar los registros que van a ser migrados y posee una estructura particular (Lencinas y Cattaneo, 2013). En las figuras 1 y 2, se representa un registro de existencias de Alephino -en formato .exp- y uno de existencias listo para ser incorporado a Koha -en formato .mrk-, luego de su transformación en un .mrc. 
FIGURA 1.

Registro de existencias tal cual fue exportado de Alephino

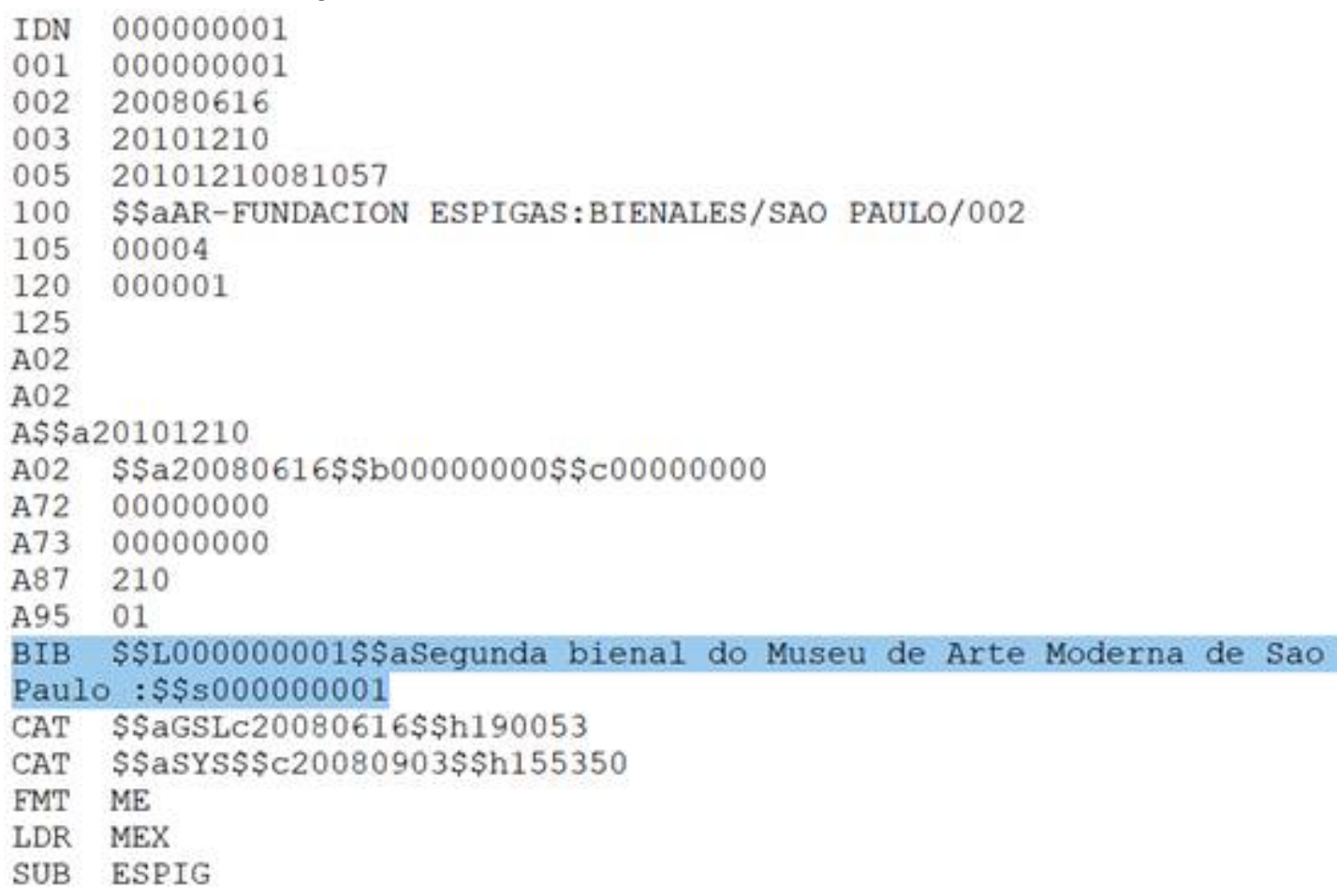

FIGURA 2

Registro formateado para ser incorporado a Koha utilizando la regla de coincidencia de registro por 001

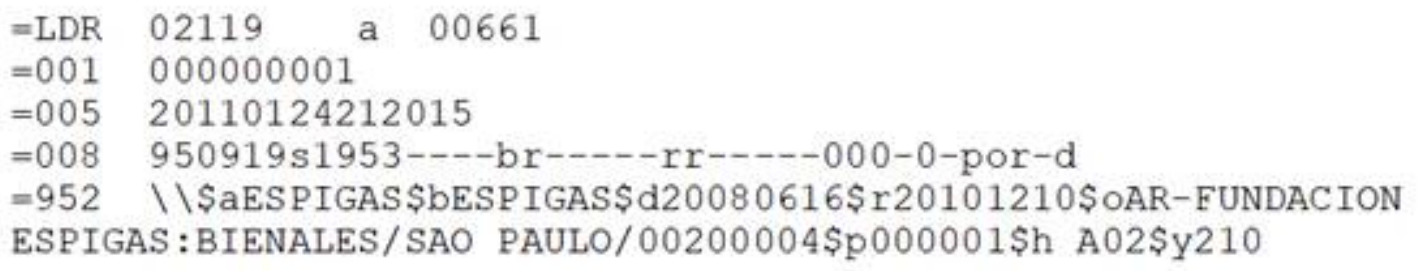

Los informes de Alephino debieron realizarse tabla por tabla, lo que significó extraer los datos bibliográficos; reformatearlos a un .mrk, para lo cual siendo tanto los archivos .exp como .mrk de texto plano simplemente se tuvo que modificar la extensión y agregarles signos igual $(=)$ a todas las líneas que contuvieran algo diferente a un salto de línea, a través de expresiones regulares simples para Textpad; convertirlos a un .mrc utilizando MaRCEdit (Chattopadhyay y Sarkar, 2017; Todd, 2018); y finalmente, incorporarlos a Koha. Asimismo, por medio de migraciones intermedias, se verificó que en las mismas no se observara pérdida de información. Dadas las diferencias en la catalogación de los recursos, debido a la diversidad de catalogadores y parámetros antes mencionada, se debió prestar una atención particular a cada registro migrado en las pruebas.

\subsection{Relación entre registros bibliográficos y de existencias}

Por otro lado, se tuvo que realizar una readecuación bastante similar -conversión de .exp en .mrk agregándole un signo igual (=) al comienzo de cada línea que contuviera caracteres- en lo que hace a los registros de existencias, con una diferencia fundamental: estos registros debían estar preparados previamente para ser 
vinculados a los bibliográficos de los cuales eran parte constitutiva. Un registro tipo de existencia tenía un 001 propio, incremental dentro de la tabla de existencias, y se vinculaba al bibliográfico por intermedio del campo propio de Alephino denominado BIB, en su subcampo "s", el cual se mencionará como BIB \$s en lo sucesivo (en la figura 1, se observa un registro de existencias tal cual fue migrado de Alephino). A partir de esto, es posible decir que a determinado 001 de un registro bibliográfico podían existir varios registros de existencias con el mismo BIB \$s, con uno solo o con ninguno. Siendo así debido a la cantidad de ítems al que un registro bibliográfico puede hacer referencia, a modo de ejemplo: un libro en dos volúmenes, del cual la biblioteca posee dos ejemplares de cada uno de ellos, poseería únicamente un registro bibliográfico, con dos registros de existencias asociados a él, vinculados por intermedio del campo 001 para el bibliográfico y el campo BIB \$s para las dos existencias relacionadas (los dos volúmenes del mismo título). A su vez, un registro bibliográfico podría vincularse a un solo registro de existencias ya sea que la biblioteca posee un documento monográfico en un volumen, o bien una obra en varias partes de la cual tiene solamente un volumen y carece $\mathrm{de} \mathrm{la/s} \mathrm{subsiguiente/s} \mathrm{parte/s,} \mathrm{y} \mathrm{en} \mathrm{ningún} \mathrm{caso} \mathrm{tiene} \mathrm{duplicados.} \mathrm{Otra} \mathrm{opción} \mathrm{puede} \mathrm{ser} \mathrm{que} \mathrm{refiera} \mathrm{a} \mathrm{ninguno,}$ por ejemplo, en el caso de un ebook, del cual se hace la descripción completa en el registro bibliográfico al cual se le añade un acceso al recurso digital, ya sea utilizando un link a una URL o la inclusión de un objeto digital dentro del sistema.

Además, para controlar que no se perdiera información en esta migración, se decidió migrar la información como parte del registro bibliográfico de Koha (en el campo 901) y en el campo reservado de Koha para ítems (952), como puede observarse en la Tabla 1.

TABLA 1

Mapeo de campos de Alephino a Koha

\begin{tabular}{|c|c|c|}
\hline Campo Koha bibliográfico & Campo Koha de ítems & Campo Alephino \\
\hline & & $=$ LDR LDR \\
\hline & & $=001$ Código auto incremental \\
\hline $901 \$ a$ & $952 \$ d$ & $=002$ Fecha de carga del ítem \\
\hline $901 \$ b$ & $952 \$ r$ & $=003$ Fecha de actualización del ítem \\
\hline $901 \$ c$ & & $=005$ Fecha y hora de actualización del ítem \\
\hline $901 \$ d$ & $952 \$ 8$ & =105 Código de colección \\
\hline $901 \$ e$ & $952 \$ 0$ & $=106$ \$aSignatura\$tTomo \\
\hline $901 \$ f$ & $952 \$ t$ & $=107$ Tomo \\
\hline $901 \$ g$ & $952 \$ t$ & $=100$ Volumen \\
\hline $901 \$ h$ & & =115 Inventario duplicado \\
\hline $901 \$ i$ & $952 \$ p$ & $=120$ Inventario \\
\hline $901 \$ \mathrm{j}$ & $952 \$ h$ & =125 Designación numérica o cronológica \\
\hline $901 \$ k$ & & =A02 Fechas de modificación inventario codificadas \\
\hline $901 \$ 1$ & & =A70 Tomo o Volumen \\
\hline $901 \$ \mathrm{~m}$ & & =A72 Fecha del número (periódicas) \\
\hline $901 \$ n$ & & =A73 Fecha de arribo esperada \\
\hline $901 \$ 0$ & & =A75 Fecha de publicación (día) \\
\hline $901 \$ p$ & & =A76 Fecha de publicación (mes) \\
\hline $901 \$ q$ & & =A82 Número \\
\hline $901 \$ r$ & & =A84 Época de periódica \\
\hline $901 \$ s$ & & =A85 Año de publicación del número \\
\hline $901 \$ t$ & $952 \$ y$ & =A87 Tipo de ítem \\
\hline $901 \$ u$ & & =A95 Status del ítem \\
\hline $901 \$ v$ & & =A97 Status de procesamiento del ítem \\
\hline $901 \$ w$ & & =BIB Título y 001 del registro bibliográfico \\
\hline $901 \$ x$ & & =CAT Catalogador \\
\hline $901 \$ y$ & & =FMT Hoja de carga bibliográfico \\
\hline $901 \$ z$ & & =SUB Código de Biblioteca \\
\hline
\end{tabular}




\subsection{Vinculando registros bibliográficos y de existencias. Modelo Koha y Alephino}

Para llevar a cabo esta correspondencia de registros se utilizó la funcionalidad de Koha denominada "Reglas de coincidencia de registro". Esta funcionalidad es un asistente de importación de registros que permite revisar si los registros a incorporarse contienen alguna coincidencia (matching en inglés) con los registros existentes en Koha y en caso de encontrarla puede sustituir totalmente (sobreescribiendo el registro bibliográfico y sus ítems), parcialmente (solamente se sustituyen / incorporan las existencias) o ignorarse si encuentra la correspondencia definida como regla. Como los registros de existencias tenían un 001 propio, autoincremental dentro de su ámbito de incidencia (cada uno de los ítems), que no era útil para vincularlo con los registros bibliográficos pero que no quería perderse para poder hacer un control de calidad postmigración, fue necesario reemplazar los 001 de los registros de existencias a un número de etiqueta MaRC21 diferente, siendo la 901 la elegida. Ello se llevó a cabo con el fin de mantener este dato del sistema integrado legado -Alephino-, a revisar en futuras comprobaciones .

Una vez completa esta tarea se utilizaron las expresiones regulares del Textpad y se identificó cada comienzo de una nueva línea que iniciara con la cadena "001" seguido de dos espacios para evitar falsos positivos, y se reemplazó cada ocurrencia con una nueva línea que comenzara con la cadena "901" seguida de dos espacios en blanco. De esta forma, cada 001 de los registros de existencias exportados de Alephino pasaron a ser 901 .

Luego se procedió a realizar una operación similar con el BIB \$s, con la diferencia de que en este caso se reconvirtió el dato para ser el nuevo -y único- 001 resultante, a fines de poder ser utilizado como elemento de correspondencia entre los registros bibliográficos y de existencias dentro del ámbito de Koha.

Además, como Alephino utiliza un esquema diferente al de Koha para guardar los datos de existencias (ver figura 1 y figura 2) fue necesario un trabajo de readecuación de los diferentes campos para integrarlos en un único 952, ya que Koha utiliza este campo en lugar del 852 para la información de existencias contenida en los registros bibliográficos. Esta tarea también se llevó a cabo usando expresiones regulares. Asimismo, una vez que se identificó a qué subcampo del 952 se correspondía cada uno de los campos de existencias de Alephino -ver tabla 1, sobre todo en lo relativo a las dos últimas columnas en las cuales se deja constancia explícita de la relación entre los campos/subcampos de Alephino y Koha- se realizaron reemplazos globales y eliminación de saltos de línea.

El mapeo de campos de existencias supuso un reto extra al no contar con documentación oficial del sistema Alephino. Para ello se recurrió a la interfaz guiada de Alephino (la interfaz de carga usual en la cual se realiza la operación diaria por parte de los bibliotecarios), de la cual se obtuvieron los nombres de las etiquetas comparando con los datos, muchas veces codificados, lo que resultó un proceso tedioso.

Como Alephino poseía datos que no serían finalmente incorporados a los registros de ítem de Koha, la solución hallada fue incorporar los mismos como nueva ocurrencia del 901 del registro bibliográfico. Ello incluía todos los campos a subcampos definidos ad-hoc, además de generar los registros de ítems de Koha utilizando el ya mencionado campo 952, transformándolo durante la migración en un nuevo registro de ítem.

\subsection{Incorporación de datos a Koha}

Una vez preparado este reformateo de datos se procedió a la conversión del .exp resultante de Alephino (ver figura 1) a .mrk y luego a .mrc (como fue explicado anteriormente) y finalmente, se definió una regla de coincidencia dentro de Koha para que se efectúe una correspondencia entre los 001 propios de los registros bibliográficos, y los nuevos registros (con datos únicamente de existencias). Mediante este proceso los registros de existencias se incorporan como ítems a los registros bibliográficos. Es por ello que los registros .mrk resultantes quedaron como puede observarse en la figura 2. 


\subsection{Control de calidad}

Finalmente, una vez verificado que el total de los registros estuviesen migrados a Koha, se accedió a la información como figuraba en Alephino y se hicieron testeos para evaluar la forma en que fue migrada a Koha. Los óptimos resultados alcanzados permitieron proceder a la instancia de capacitación del personal en el sistema nuevo tanto en lo relacionado a catalogación como en los diversos módulos que contempla, a fin de hacer un uso eficiente del mismo.

\section{Conclusiones}

La migración de Alephino a Koha llevada a cabo por la biblioteca Espigas tuvo algunos elementos diferenciadores de lo reseñado en la bibliografía impresa y web: ya sea por las propias características del software Alephino (diferente al Aleph, del cual existen trabajos documentando el proceso), o por alguna cuestión relativa a la versión y/o adquisición de módulos de este sistema integrado de gestión bibliotecaria privativo. Lamentablemente estas cuestiones no pudieron ser resueltas vía el soporte técnico oficial, del cual, si bien estaba contratado, no se obtuvo respuesta útil al objetivo. Los inconvenientes que tuvieron que sobrellevarse podrían resumirse en:

- No existen trabajos en las que se dé cuenta de una migración del sistema Alephino a Koha.

- No hubo forma de conseguir una respuesta clara y/o útil en algunos casos de parte del proveedor del sistema Alephino, esto es un elemento muy complicado de sobrellevar, ya que para realizar una migración debe conocerse lo más posible la estructura de los datos del sistema actual, así como del sistema al que serán trasladados, siendo software privativo es imposible poder estudiar los elementos constitutivos de un sistema sin intervención de la empresa proveedora.

- La migración de datos de esta versión de Alephino no proveía forma de extraer los datos bibliográficos y de existencias relacionados entre sí en un mismo archivo diferenciando los elementos a través de etiquetas 852,952 , etc.

Por otro lado, a partir de esta experiencia, y en base a lo relevado en la bibliografía especializada (Todd, 2018), se puede aseverar que los bibliotecarios tienen competencias para afrontar los procesos relativos a la migración de datos. A nuestro entender, la mejor forma de hacerlo es en base a una agenda o programa que responda a un ambiente colaborativo, aunque sea en lo respectivo a los profesionales de la misma disciplina (el caso de nuestra colega de Di Tella auxiliando desde afuera del grupo en forma ad-honorem es un muy buen ejemplo) si no se cuenta o necesitan otros recursos, como también a un contexto de aprendizaje. De este modo el presente artículo pretende ser una contribución a la comunidad de Koha en particular, y a la bibliotecaria toda en general.

A su vez, estas dos características son consideradas impensables para un proyecto de software privativo, en el cual participan un detentador de datos/conocimiento y procesos, y unos clientes que reciben el fruto de ese producto, sin tener este último participación ni conocimiento respecto a una información que le compete; de la cual no le reportará ningún conocimiento más allá de su uso con la herramienta a través de la interfaz guiada. Es en este sentido nos parece importante promover el uso del software libre, un camino que redunda en conocimiento, alimentando un ciclo virtuoso a través del cual los bibliotecarios pueden desarrollarse cada vez más plenamente desde lo profesional. 


\section{Referencias bibliográficAs}

Chattopadhyay, S., y Sarkar, A. (2017). Bibliographic Data Migration from LibSys to Koha\#: Experience at St. Xavier's College, Kolkata (pp. 159-167). Bengal Library Association, Kolkata. Recuperado de http://eprints.rclis.org/3 $1688 /$

How to migrate from Aleph to Koha: ALEPH 21 to Koha 3.22 migration scripts. (s. f.). Recuperado de https://mypag es.lib.ltu.se/AlephToKohaMigrationScriptsInEnglish.html

Kohn, K., y McCloy, E. (2010). Phased Migration to Koha: Our Library's Experience, Journal of Web Librarianship, 4(4), 427-434. https://doi.org/10.1080/19322909.2010.485944

Lencinas, V., y Cattaneo, A.R. (2013). Migración de registros bibliográficos a Marc21. Problemáticas, técnicas y experiencias en torno a la implementación del sistema Digibepé (Koha) de Conabip. Asociación de Bibliotecarios de Córdoba. Recuperado de http://eprints.rclis.org/19908/

Official Website of Koha Library Software. (s. f.). Recuperado de https://koha-community.org/

Rus Mata, S.,y Plaza, M. A. (2017). Repositorio Institucional de la Universidad Torcuato Di Tella\#: migración de tesis. Ponencia presentada en 49va Reunión Nacional de Bibliotecarios, 25 al 27 de abril de 2017, Buenos Aires, Argentina. Recuperado de https://repositorio.utdt.edu/handle/utdt/10862

Todd, C. R. (2018). Librarian as data migrator: a functional pathway from Millennium to Koha. Digital Library Perspectives, 34(1), 60-69. https://doi.org/10.1108/DLP-09-2017-0035

\section{Notas}

1 En Kohn y McCloy (2010) se describe algo similar, al igual que en Todd (2018), ya que evidentemente acceder a la base de datos en un sistema privativo no es lo deseable por parte del desarrollador.

2 Cabe resaltar que en las migraciones no se recomienda descartar datos, lo que ya fue exportado puede ser reutilizado a futuro, aunque en el momento no se tenga precisión de cómo. 